\title{
Insegurança alimentar e nutricional em famílias do meio rural brasileiro: revisão sistemática
}

\author{
Food and nutritional insecurity in families in the Brazilian rural \\ environment: a systematic review
}

Paula Torres Trivellato ${ }^{1}$

Dayane de Castro Morais ${ }^{1}$

Sílvia Oliveira Lopes ${ }^{1}$

Elizangela da Silva Miguel ${ }^{1}$

Sylvia do Carmo Castro Franceschini ${ }^{1}$

Silvia Eloiza Priore ${ }^{1}$

${ }^{1}$ Departamento de Nutrição e Saúde, Centro de Ciências Biológicas e da Saúde, Universidade Federal de Viçosa. Av. P. H. Rolfs, Campus Universitário. 36570-900 Viçosa MG Brasil.paulatrivellato@ hotmail.com

\begin{abstract}
This review sought to analyze food and nutritional insecurity in the Brazilian rural environment, and the measurement instruments used to assess this situation. A systematic review was conducted in the SciELO, PubMed and CAPES databases, using the search terms: 'food security' and 'rural,' Subsequently, a reverse search was performed in the 12 articles selected. The inclusion criteria were: original articles related to food and nutritional (in)security in the Brazilian rural population. Twelve studies were included. Most of them used the Escala Brasileira de Insegurança Alimentar - EBIA (Brazilian Scale for Food Insecurity) and socioeconomic questionnaires to assess food insecurity (FI), an association being found between them. Five studies addressed the nutritional aspect by anthropometry but found no association with FI. Food consumption was assessed by four studies and an association was detected. The results show a high prevalence of FI in rural areas in relation to the population analyzed in the Pesquisa Nacional por Amostra de Domicílio PNAD (National Household Sample Survey) in the years 2009 and 2013, as well as a high prevalence of severe FI. The conclusion reached is that the rural environment is vulnerable, mainly regarding food and nutritional issues and the associated socioeconomic determinants.
\end{abstract}

Key words Food security, Rural
Resumo Esta revisão objetivou analisar a insegurança alimentar e nutricional no meio rural brasileiro, bem como os instrumentos de mensuração utilizados na avaliação dessa situação. Realizou-se revisão sistemática nas bases de dados SciELO, Pubmed e o portal de periódicos da CAPES, usando os termos de busca: 'segurança alimentar' e 'rural'. Posteriormente foi realizada busca reversa dentro dos artigos selecionados. Os critérios de inclusão foram: artigos originais, relacionados à (in) segurança alimentar e nutricional na população rural brasileira. Foram incluídos 12 estudos. A maioria utilizou a Escala Brasileira de Insegurança Alimentar-EBIA e questionários socioeconômicos para avaliar a insegurança alimentar (IA), sendo encontrada associação entre eles. Cinco estudos abordaram o aspecto nutricional pela antropometria, porém não encontraram associação com a IA. O consumo alimentar foi avaliado por quatro estudos e foi observada associação. Os resultados mostram alta prevalência de IA no meio rural em relação à população analisada na Pesquisa Nacional por Amostra de Domicílio -PNAD nos anos de 2009 e 2013, assim como alta prevalência de IA grave. Conclui-se que o meio rural se apresenta em vulnerabilidade, principalmente quanto à questão alimentar e nutricional e aos determinantes socioeconômicos atrelados.

Palavras-chave Segurança alimentar, Rural 


\section{Introdução}

O conceito de Segurança Alimentar e Nutricional vem sendo construído e evolui em consonância à evolução humana e aos interesses e relações sociais, passando por alterações em função da própria História. O termo segurança alimentar vem sendo debatido desde a $1^{\circ}$ Guerra Mundial, passando ao longo dos anos por diferentes conotações e englobando outras dimensões até expandir para segurança alimentar e nutricional ${ }^{1}$.

O termo Segurança Alimentar e Nutricional (SAN), adotado no território brasileiro, é definido pela Lei no 11.346/2006 como "a garantia de acesso regular e permanente a alimentos de qualidade, em quantidade suficiente, sem comprometer o acesso a outras necessidades essenciais e que respeitem a diversidade cultural, sendo ambiental, cultural, econômica e socialmente sustentáveis ${ }^{1}$ e tendo como princípios básicos o direito humano à alimentação adequada e à soberania alimentar" ${ }^{2-4}$.

Para alcançar SAN fica implícito a necessidade de condições socioeconômicas e qualidade de vida adequadas. A Insegurança Alimentar e Nutricional (IAN) pode ser vista tanto em situações de alimentação insuficiente, como fome e desnutrição, quanto em situações de doenças decorrentes da alimentação inadequada, como o excesso de peso e carências específicas. As consequências dessas situações recaem sobre diferentes setores, mais diretamente o de saúde ${ }^{5-7}$.

Para o planejamento de programas e políticas públicas de cunho preventivo, de promoção da saúde e de combate à fome, há de se considerar a situação de (in)segurança alimentar e nutricional e para isso é preciso que haja indicadores desta situação. O único método disponível para a medida direta da insegurança alimentar (IA) é a Escala Brasileira de Insegurança Alimentar (EBIA), que aborda e classifica a percepção da família sobre a alimentação nos últimos três meses por meio de quatorze perguntas estruturadas ${ }^{8,9}$.

A (in)segurança alimentar e nutricional também pode ser avaliada por indicadores nutricionais e socioeconômicos, uma vez que possui múltiplas dimensões, porém é válido ressaltar que cada indicador tem sua ótica, tornando-os complementares, e o uso combinado destes é importante para entender e inferir sobre a situação ${ }^{7,9}$.

Na população brasileira a IA é mais prevalente no meio rural, como mostram a Pesquisa Nacional por Amostra de Domicílio (PNAD) realizadas em 2004, 2009 e 2013 ${ }^{10-12}$ e também a Pes- quisa Nacional de Demografia e Saúde (PNDS) $2006^{13}$. Tais inquéritos utilizaram a EBIA para mensurar a IA. O meio rural apresenta níveis de pobreza elevados, combinados a baixa escolaridade e desenvolvimento, que contribuem para a instalação deste quadro ${ }^{4,7}$.

Em virtude da situação de vulnerabilidade do meio rural brasileiro faz-se necessário conhecer o perfil de IAN deste segmento da população e como este perfil tem sido avaliado, a fim de inferir sobre os determinantes e levantar possíveis ações de enfrentamento do quadro. Esta revisão objetivou analisar a insegurança alimentar e nutricional no meio rural brasileiro, bem como os instrumentos de mensuração utilizados na avaliação dessa situação.

\section{Metodologia}

Trata-se de uma revisão sistemática norteada pelo questionamento: "Qual a situação de (in) segurança alimentar e nutricional na população rural brasileira e como esta situação é medida?". A revisão foi realizada em pares seguindo as recomendações PRISMA (Preferred Reporting Items for Systematic Reviews and Meta-Analysis) ${ }^{14}$.

Realizou-se busca para seleção dos estudos nas bases de dados Scientific Electronic Library Online-SciELO e US National Library of Medicine National Institutes of Health-Pubmed, e no Portal de Periódicos da Coordenação de Aperfeiçoamento de Pessoal de Nível Superior (CAPES), usando os termos de busca: 'segurança alimentar' e 'rural'. Não se utilizou o termo "segurança alimentar e nutricional" para não limitar a busca, visto que o único instrumento direto desta situação mensura apenas a dimensão alimentar da insegurança.

Os critérios de inclusão foram: artigos originais, relacionados ao tema de interesse desse estudo. Excluiu-se artigos de revisão, resumos de congressos, monografias, dissertações, teses, capítulos de livro, assim como estudos realizados em populações internacionais, indígenas e quilombolas e estudos que não classificaram separadamente o meio rural do urbano quanto a (in) segurança alimentar. Não foi estabelecido o ano de publicação como critério.

Para elaboração da revisão sistemática avaliou-se inicialmente os títulos, seguido da leitura dos resumos e posteriormente a leitura na íntegra dos estudos. Identificou-se 103 artigos originais nas buscas realizadas. Foram excluídos 73 artigos após a leitura dos títulos e 9 por serem repetidos. 
Em seguida excluiu-se pela leitura dos resumos 11 artigos, sendo selecionados 10 para leitura na íntegra, sendo todos incluídos.

Posteriormente foi realizada busca reversa nos 10 artigos incluídos, buscando pelos termos dentro das listas de referência dos estudos a fim de identificar artigos originais publicados, não localizados inicialmente, e que atendessem aos critérios de inclusão. Foram identificados e incluídos 2 estudos, totalizando assim 12 artigos para essa revisão.

O esquema representativo da busca e número de estudos encontrados e elegíveis, está representado na Figura 1.

\section{Resultados}

Os estudos utilizados nessa revisão foram publicados no período de 2008 a 2017. Quanto à caracterização das amostras dos estudos selecionados, todos trabalharam com famílias rurais, houve predominância da região Nordeste $60 \%$ ( $\mathrm{n}=$ 6) ${ }^{15-20}$ e presença de $33,3 \%(n=4)^{4,20-22}$ de estudos com grupos relacionados ao movimento sem terra. A síntese dos principais dados dos artigos em estudo está representada no Quadro 1.

Dentre os estudos $(n=12)$ predominou $(n=$ 11) a aplicação da EBIA como método de avaliação da IA na população, utilizada sempre em conjunto a outros métodos de avaliação indireta ${ }^{15-25}$.

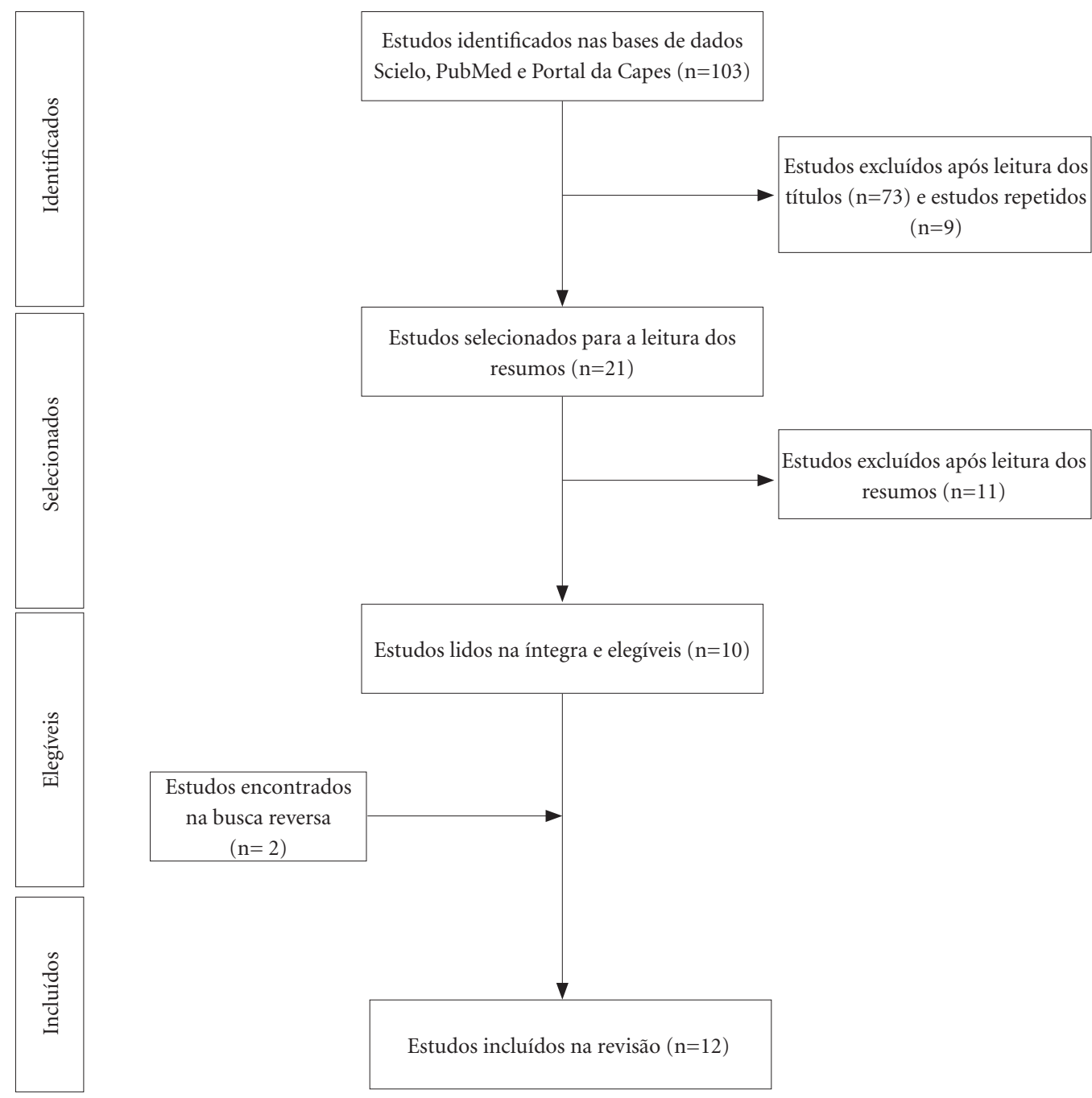

Figura 1. Esquema de busca e seleção de estudos incluídos na revisão sistemática. 


\begin{tabular}{|c|c|c|c|c|c|}
\hline & 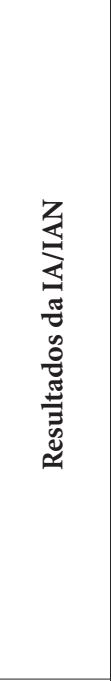 & 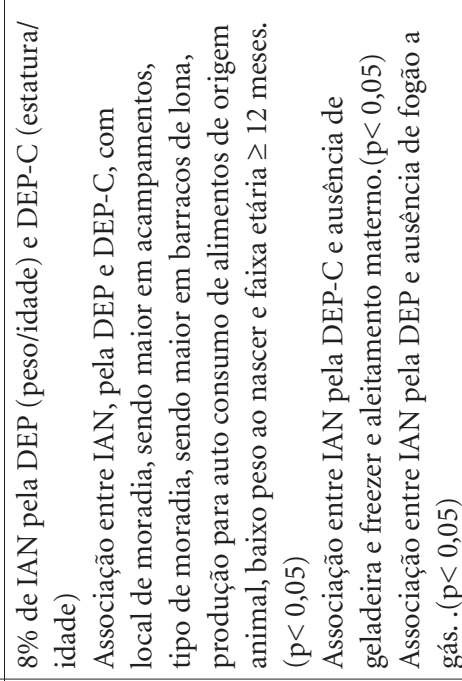 & 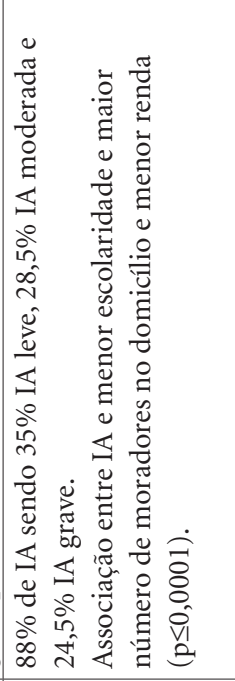 & 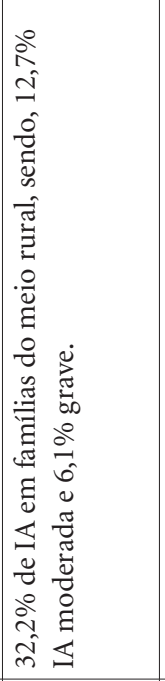 & 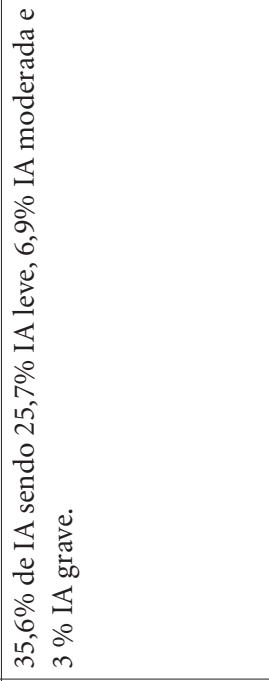 \\
\hline & 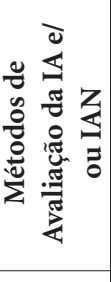 & 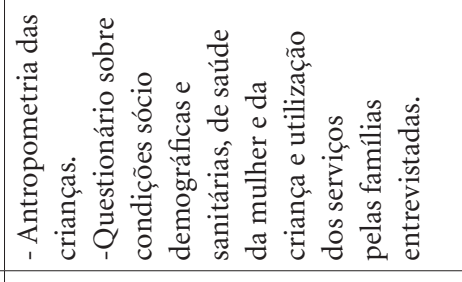 & 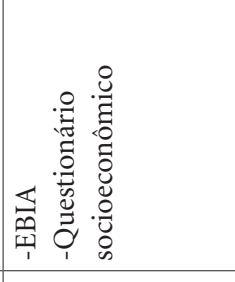 & 死 & 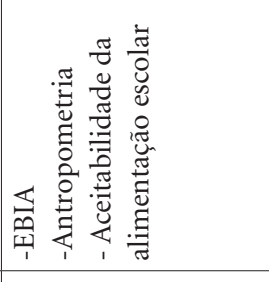 \\
\hline 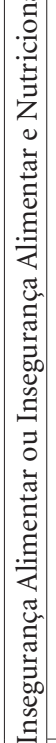 & 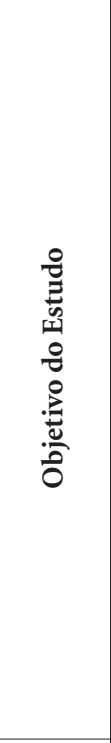 & 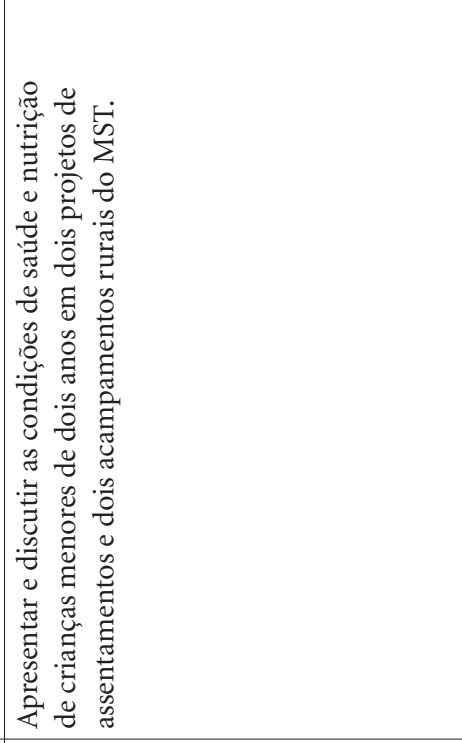 & 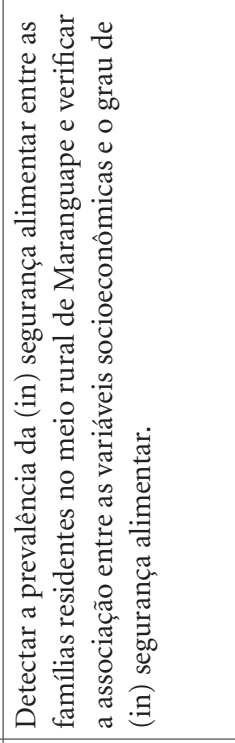 & 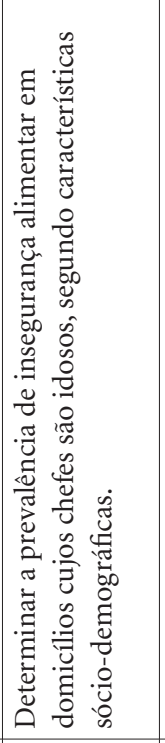 & 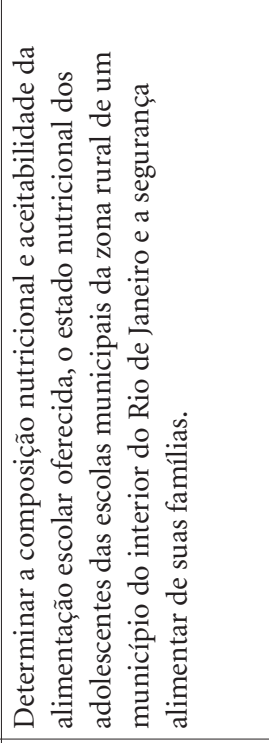 \\
\hline 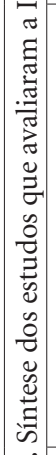 & 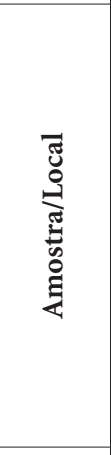 & 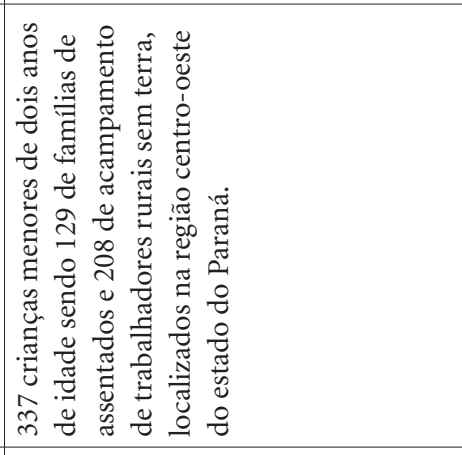 & 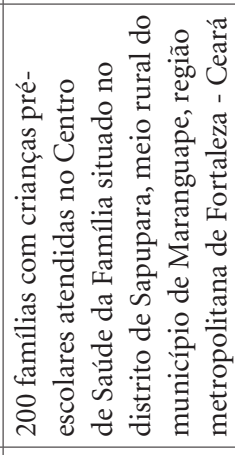 & 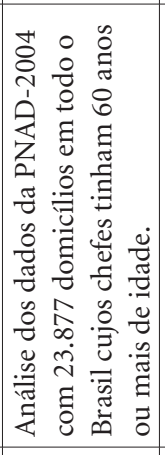 & 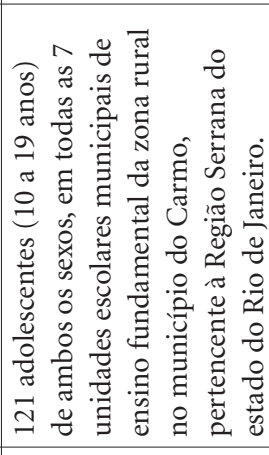 \\
\hline & 要定 & 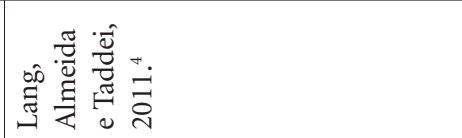 & 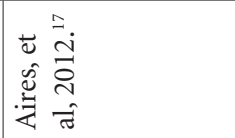 & 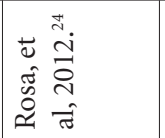 & 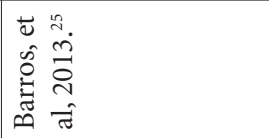 \\
\hline
\end{tabular}




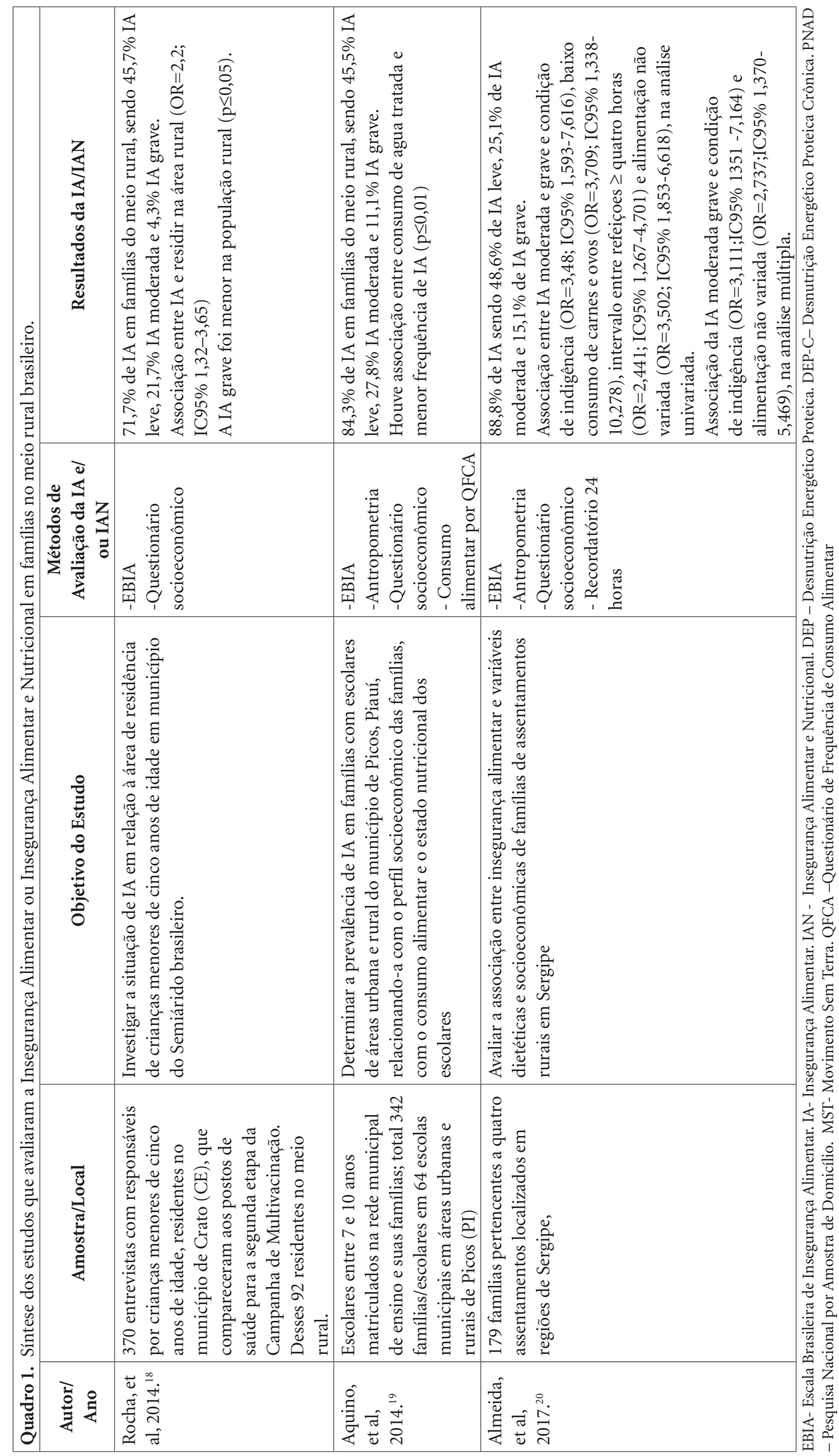


Questionário socioeconômico e demográfico foi o método de avaliação indireto mais utilizado, estando presente em $66,6 \%(n=8)$ dos trabalhos $^{4,15,17-19,21-23}$, sendo encontrada associação com a IA em todos estes. Observou-se associação entre IA e menor renda ${ }^{15,17,20,23}$; produção insuficiente de alimentos ${ }^{4,15}$; escolaridade ${ }^{17}$; endividamento ${ }^{15}$; número de moradores no domicílio maior que cinco $^{17}$; ausência de água tratada ${ }^{19}$; local de moradia $^{4,18,22}$; e tipo de moradia ${ }^{4}$. Quanto ao local de moradia, a IA foi maior em acampados em relação aos assentados ${ }^{4}$; no bairro dos boias frias em relação aos assentados e acampados ${ }^{22}$; e em área rural quando comparada à urbana, sendo que famílias que residiam em área rural apresentavam duas vezes mais chance de $\mathrm{IA}^{18}$. Ao considerar o tipo de moradia, observou-se que a IA era mais prevalente entre os residentes em barracos de lona ${ }^{4}$.

A antropometria, como indicador indireto de IA, foi utilizada por $41,6 \%(n=5)$ dos estu$\operatorname{dos}^{4,16,19,20,25}$. Entre esses, $60 \%(n=3)$ fizeram avaliação antropométrica somente em crianças $^{4,16,19}$, $20 \%(\mathrm{n}=1)$ em adolescentes ${ }^{25}$, e $20 \%(\mathrm{n}=1)$ realizaram antropometria em todos os membros da família, abarcando todas as faixas etárias ${ }^{19}$. Em relação aos índices, os estudos avaliaram peso por idade ${ }^{4,16,19}$, estatura por idade $e^{4,16,19,25}$ e índice de massa corporal por idade ${ }^{4,16,19,20,25}$. Nenhum dos estudos encontrou associação entre estado nutricional (excesso de peso, baixo peso e/ou desnutrição crônica) e IA.

O consumo alimentar também foi utilizado como método indireto sendo relacionado a IA em $33,3 \%(n=4)$ dos estudos ${ }^{19,20,23,25}$. Entre as metodologias para mensurar o consumo, utilizou-se Questionário de Frequência de Consumo Alimentar (QFCA) ${ }^{19,23}$, recordatório 24 horas $^{20} \mathrm{e}$ aceitabilidade da merenda escolar ${ }^{25}$. Observou-se associação entre IA e consumo de verduras e su$\cos$ de frutas, sendo a insegurança maior entre famílias com consumo menor que cinco vezes por semana desses alimentos ${ }^{23}$. Quanto à variedade da dieta, indivíduos com alimentação não variada apresentavam 2,7 vezes mais chances de IA $^{20}$. Embora sem diferença estatística, o consumo de carne e vegetais foi maior entre crianças em situação de segurança alimentar em um estudo ${ }^{19}$. Não houve diferença em relação a aceitabilidade da alimentação escolar e IA ${ }^{25}$.

A IA, pela EBIA, variou de $32,2 \%$ a $88,8 \% 15-21$, ${ }^{23-25}$, e a IA grave de $3 \%$ a $39,5 \% 0^{15-20,22-25}$. Somente um estudo não utilizou a EBIA ${ }^{4}$. Este avaliou IAN dada pelo resultado da antropometria em crianças, indicando 8\% de insegurança pela desnutri- ção energético proteica ou desnutrição crônica, a relacionando com as condições de saúde e moradia em famílias de trabalhadores rurais sem terra assentados e em acampamentos.

Entre os estudos, 50\% $(\mathrm{n}=6)^{4,15,19,20,22,23,25}$ utilizaram indicadores de IAN, abordando a dimensão nutricional da insegurança por meio da avaliação do estado nutricional e do consumo alimentar. Porém, somente Lang et al. ${ }^{4}$, Almeida et al..$^{20}$ e Carneiro et al. ${ }^{22}$ inferiram sobre a situação de IAN utilizando estes indicadores.

\section{Discussão}

A (in)segurança alimentar e nutricional apresenta uma abrangência conceitual e uma complexidade de fatores determinantes, por isso se torna tão desafiador mensurar tal condição, devendo ser utilizados indicadores da dimensão alimentar e da dimensão nutricional2 ${ }^{26,27}$.

Em relação à dimensão alimentar, a EBIA é o único indicador direto e o mais utilizado na literatura para avaliar essa situação. Nesta revisão todos os estudos que avaliaram IA pela EBIA apresentaram valores maiores que $35,2 \%$ e 35,3 $\%$, encontrados pelas PNAD $2009^{11}$ e $2013^{12}$ para domicílios do meio rural, exceto Rosa et al..$^{24}$ que encontraram $32,2 \%$ de IA em domicílios rurais chefiados por idosos.

Quando analisada a situação de IA grave, que se refere à restrição quantitativa de alimentos nos últimos três meses, a maioria dos estudos ${ }^{15-17,19-24}$ apresentou valores de IA grave acima de 5,5\%, que foi o valor encontrado pela PNAD 2013 12 para a população rural. Esses resultados podem ser devido à maioria dos estudos desta revisão ter sido realizada em populações rurais localizadas em áreas de maior vulnerabilidade, principalmente na região Nordeste. Este fato é relevante ao se considerar que 59\% do total de 16 milhões de pessoas em condição de extrema pobreza no Brasil, em 2010, estão localizados na região Nordeste, com destaque para Alagoas, Piauí, Paraíba e Pernambuco ${ }^{28}$. Vale destacar que a Paraíba foi cenário de dois estudos ${ }^{15,16}$ analisados nessa revisão e o Piauí de um ${ }^{19}$.

Embora a EBIA seja um instrumento validado tanto para a população urbana quanto para a rural, ela não tem objetivo de fazer menção à possibilidade de produção agrícola para autoconsumo e nem práticas de cultivos coletivos e trocas de alimentos como formas de acesso e promotoras de segurança alimentar. Também não objetiva considerar a posse de terra, acesso 
à água ou a insumos agrícolas no meio rural ${ }^{8}$. A mesma condiciona o acesso ao alimento à renda, sendo que no meio rural essa condição pode ser diferente. Portanto, para uma abordagem mais completa da IA, faz-se necessário aprofundar em fatores socioeconômicos e de acesso a bens e serviços, levando a uma compreensão mais abrangente, condizente com a pluralidade da questão ${ }^{29}$.

A posse de terra tem sido atribuída ao possível sustento da família e produção de alimentos para autoconsumo ${ }^{4,15,22}$, contribuindo para SAN. Carneiro et al. ${ }^{22}$ encontraram que $57 \%$ das famílias de assentados conseguiam viver apenas da produção do lote o que as diferenciavam dos acampados e bóias-frias, assim como Busato et al. ${ }^{21}$ que encontraram, na localidade rural estudada, que a maioria dos alimentos consumidos pelas famílias advinha da própria produção, afirmando a relevância da posse da terra e da produção para autoconsumo na promoção da SAN. Outros autores, não incluídos nesta revisão, também observaram que o acesso à terra e à possibilidade de plantio para autoconsumo, contribuem para melhorias nas condições de alimentação ${ }^{30}$.

Apenas $u^{4}$ dos estudos incluídos nessa revisão considerou a presença de desnutrição como indicador de IAN. Embora alguns estudos ${ }^{16,19,20,25}$ tenham utilizado a antropometria, estes não classificaram a insegurança pela presença de distrofia nutricional. Nesta revisão, nenhum dos estudos encontraram associação entre o estado nutricional e a IA pela EBIA, porém a literatura tem apresentado associação da IA com obesidade ${ }^{31}$, baixa estatura $^{32,33}$ e baixo peso ${ }^{34}$, sem restrição quanto ao local de moradia.

Os estudos desta revisão, em sua maioria, utilizaram dados socioeconômicos e demográficos em complementação à análise de IA dada pela EBIA. A baixa renda esteve associada à IA, podendo essa relação estar atribuída ao fato de que a EBIA está atrelada a questões de renda. As demais condições socioeconômicas e demográficas, como acesso a bens e serviços, escolaridade, tipo e condições de moradia, quando precárias, estão condicionadas à baixa renda e consequentemente a situação de IA. Essas condições quando presentes no meio rural se intensificam em função da pobreza, baixos índices de educação e desenvolvimento e má distribuição de terra, corroborando com a instalação da IAN neste público ${ }^{3}$.

A IA no meio rural tem-se mostrado mais prevalente em relação ao urbano, como demonstrado pela $\mathrm{PNAD}^{10-12}$. Esses resultados podem ser atribuídos às maiores dificuldades encontradas no desenvolvimento das políticas públicas de saúde e saneamento no meio rural. O processo de modernização da agricultura no Brasil, excludente a muitos produtores rurais, também contribui, uma vez que amplia a vulnerabilidade socioambiental dessa população ${ }^{22}$.

Em relação ao consumo alimentar, observouse menor ingestão de frutas, verduras, carnes e ovos, maior intervalo entre as refeições e alimentação não variada associados à presença de IA $^{19,20,23,24}$. A alimentação monótona, reduzida em micronutrientes e proteína, pode estar associada à renda familiar insuficiente, refletindo nas escolhas alimentares não saudáveis características da situação de IAN $^{26,35,36}$. Ressalta-se que nenhum dos estudos avaliou a ingestão de nutrientes e energia e sim relacionaram a SAN a grupos de alimentos.

O baixo consumo de frutas, vegetais e carnes magras presente nas famílias residentes do meio rural $^{11,37}$ as tornam mais vulneráveis em relação à dimensão nutricional da insegurança, podendo levar a inadequação do estado nutricional e fome oculta $^{38}$.

$\mathrm{Na}$ avaliação da IAN faz-se necessário a utilização de métodos complementares à EBIA com $\mathrm{o}$ intuito de abranger as dimensões alimentar e nutricional desta situação $0^{26,27}$. Portanto destacase a importância de relacionar os diferentes indicadores, de ambas as dimensões da IAN, e não apenas considerá-los de forma isolada.

Os estudos incluídos nesta revisão sistemática apresentaram como limitações o fato de todos serem transversais, não permitindo relação causal; não avaliação da dimensão nutricional da insegurança ${ }^{15,17,18,22}$, uma vez que utilizaram apenas a EBIA e indicadores socioeconômicos. Destaca-se também a não associação entre os diferentes indicadores da IAN, mesmo estes tendo sido coletados ${ }^{16,18,21}$. A EBIA ter sido enviada aos pais dos adolescentes e não aplicada por entrevistador treinado ${ }^{25}$ e junção das situações de segurança alimentar e insegurança alimentar leve $e^{20}$ nas análises são pontos negativos para obtenção de resultados mais fidedignos. Outra limitação identificada foi a utilização de índice antropométrico não recomendado para avaliação de crianças acima de cinco anos, como o índice peso/idade, principalmente em estudo transversal ${ }^{19}$.

\section{Considerações finais}

Os resultados expõem a situação vulnerável no meio rural, principalmente quanto à questão alimentar e nutricional e aos determinantes so- 
cioeconômicos atrelados, e indica a pertinente necessidade de ações voltadas a esse público.

A SAN, por ser multifacetada, necessita de abordagem metodológica variada e complementar. A EBIA tem sido utilizada por ser o único método de avaliação direta da IA, porém essa escala não contempla a dimensão nutricional, fazendose necessária a utilização de outros métodos.

\section{Colaboradores}

PT Trivellato, DC Morais, SO Lopes e ES Miguel trabalharam na elaboração, pesquisa, redação, correção e edição final. SCC Franceschini e SE Priore participaram na redação, revisão crítica e edição final.
A temática abordada nesta revisão busca trazer o debate sobre a IAN no meio rural e despertar a atenção a essa importante parcela da população brasileira, que muitas vezes é marginalizada. Pretende-se contribuir com um referencial científico e somar informações para que medidas de avaliação e promoção da segurança alimentar possam ser efetivas.

\section{Referências}

1. Burity V, Franceschini T, Valente F, Recine E, Leão M, Carvalho MF. Direito humano à alimentação adequada no contexto da segurança alimentar e nutricional. Brasília: Abrandh; 2010.

2. Brasil. Lei $\mathrm{n}^{\circ} 11.346$, de 15 de setembro de 2006. Cria o Sistema Nacional de Segurança Alimentar e Nutricional - SISAN com vistas em assegurar o direito humano à alimentação adequada e dá outras providências. Diário Oficial da União 2006; 18 set.

3. Burlandy L. A construção da política de segurança alimentar e nutricional no Brasil: estratégias e desafios para a promoção da intersetorialidade no âmbito federal de governo. Cien Saude Colet 2009; 14(3):851-860.

4. Lang RMF, Almeida CCB, Taddei JAAC. Segurança alimentar e nutricional de crianças menores de dois anos de famílias de trabalhadores rurais Sem Terra. Cien Saude Colet 2011; 16(7):3111-3118.

5. Segall-Corrêa AM, Marin-Léon L, Helito H, Pérez-Escamilla R, Santos LPM, Paes-Sousa R. Transferência de renda e segurança alimentar no Brasil: análise dos dados nacionais. Rev Nutr 2008; 21(Supl.):39-51.

6. Alves KPS, Jaime PC. A Política Nacional de Alimentação e Nutrição e seu diálogo com a Política Nacional de Segurança Alimentar e Nutricional. Cien Saude Colet 2014; 19(11):4331-4339.

7. Morais DC, Dutra LV, Franceschini SDCC, Priore SE. Insegurança alimentar e indicadores antropométricos, dietéticos e sociais em estudos brasileiros: uma revisão sistemática. Cien Saude Colet 2014; 19(5):1475-1487.

8. Nascimento AL, Gonçalves FCLSP, Maia SR, Schneider S, Liras P. A construção de capacidades e meios de vida na garantia da segurança alimentar do rural pernambucano. Rev Segurança Alimentar e Nutricional 2012; 19(2):50-62.

9. Pedraza DF, Gama JSDFA. Segurança alimentar e nutricional de famílias com crianças menores de cinco anos do município de Campina Grande, Paraíba. Rev Bras Epidemiol 2015; 18(4):906-917.

10. Instituto Brasileiro de Geografia e Estatística (IBGE). Pesquisa Nacional por Amostra de Domicílios (PNAD). Segurança Alimentar: 2004. Rio de Janeiro: IBGE; 2006.

11. Instituto Brasileiro de Geografia e Estatísticas (IBGE). Pesquisa Nacional por Amostra de Domicílios (PNAD). Segurança Alimentar: 2009. Rio de Janeiro: IBGE; 2010. 
12. Instituto Brasileiro de Geografia e Estatísticas (IBGE). Pesquisa Nacional por Amostra de Domicílios (PNAD). Segurança Alimentar: 2013. Rio de Janeiro: IBGE; 2014.

13. Brasil. Ministério da Saúde (MS). Pesquisa Nacional de Demografia e Saúde da Criança e da Mulher - PNDS 2006: dimensões do processo reprodutivo e da saúde da criança. Brasília: MS; 2009.

14. Galvão TF, Pansani TDSA, Harrad D. Principais itens para relatar Revisões sistemáticas e Meta-análises: A recomendação PRISMA. Epidemiologia e Serviços de Saúde 2015; 24(2):335-342.

15. Vianna RPT, Segall-Corrêa AM. Insegurança alimentar das famílias residentes em municípios do interior do estado da Paraíba, Brasil. Rev Nutr 2008; 21(Supl.):111122.

16. Oliveira JS, Lira PIC, Andrade SLLS, Sales AC, Maia SR, Batista Filho M. Insegurança alimentar e estado nutricional de crianças de São João do Tigre, no semi-árido do Nordeste. Rev Bras Epidemiol 2009; 12(3):413-423.

17. Aires JS, Martins MC, Joventino ES, Ximenes LB. (in) segurança alimentar em famílias de pré-escolares de uma zona rural do Ceará. Acta Paul Enferm 2012; 25(1):102-108.

18. Rocha BM, Lima RT, Almeida PC. Insegurança alimentar relacionada à área de residência em município do Semiárido brasileiro. Cad. Saúde Colet. 2014; 22(2):205-211.

19. Aquino JS, Sequeira-de-Andrade LS, Silvia PEBA, Silva AP, Vieira CRS, Lira PIC. Food insecurity and socioeconomic, food and nutrition profile of schoolchildren living in urban and rural areas of Picos, Piauí. Rev. Nutr. 2014; 27(4):395-404.

20. Almeida JA, Santos AS, Nascimento MAO, Oliveira JVC, Silva DG, Mendes-Neto RS. Fatores associados ao risco de insegurança alimentar e nutricional em famílias de assentamentos rurais. Cien Saude Colet 2017 22(2):479-488.

21. Busato MA, Gallina LS, Dreyer DC, Quadros JC, Lavratti E, Teo CRPA. Segurança alimentar e nutricional e as condições do ambiente em assentamento rural de Santa Catarina. Alimentos e Nutrição Araraquara 2011; 22(4):555-559.

22. Carneiro FF, Tambellini AT, Silva JAD, Haddad JPA, Búrigo AC, Sá WRD, Bertolini VA. Saúde de famílias do Movimento dos Trabalhadores Sem Terra e de bóiasfrias, Brasil, 2005. Rev Saude Publica 2008; 42(4):757763.

23. Nobre LN, Murta NMG, Souza MM, Ferreira NC, Cardoso LM, Hamacek FR. Segurança Alimentar em uma Comunidade Rural no Alto Vale do Jequitinhonha/MG. Rev Segurança Alimentar e Nutricional 2009; 16(1):18-31.

24. Rosa TEDC, Mondini L, Gubert MB, Sato GS, Benício MHDA. Segurança alimentar em domicílios chefiados por idosos, Brasil. Rev Bras Geriatr Gerontol 2012; 15(1):69-77.

25. Barros MS, Fonseca VM, Meio MDBB, Chaves CR. Excesso de peso entre adolescentes em zona rural e a alimentação escolar oferecida. Cad. Saúde Colet 2013; 21(2):201-208.
26. Segall-corrêa AM. Insegurança alimentar medida a partir da percepção das pessoas. Estudos Avançados 2007; 21(60):143-154.

27. Kepple AW, Segall-Corrêa AM. Conceituando e medindo segurança alimentar e nutricional. Cien Saude Colet 2011; 16(1),187-199.

28. Ottonelli J, Mariano JL. Pobreza multidimensional nos municípios da Região Nordeste. Rev Administração Pública 2014; 48(5):1253-1280.

29. Takagi M, Silva JG, Grossi MD. Pobreza e Fome: em busca de uma metodologia para quantificação do problema no Brasil [texto para discussão]. Campinas: Unicamp; 2001.

30. Leite SP. Impacto dos Assentamentos: um estudo sobre o meio rural brasileiro. São Paulo: Editora UNESP; 2004.

31. Valásquez-Melendez G, Schlussel MM, Brito AS, Silva AAM, Lopes-Filho J, Kac G. Mild but not light or severe food insecurity is associated with obesity among brazilian women. J Nutr 2011; 141(5):898-902.

32. Oliveira JS, Lira PIC, Veras ICL, Maia SR, Lemos MCC, Andrade SLLS, Viana Junior MJ, Pinto FCL, Leal VS, Batista Filho M. Estado nutricional e insegurança alimentar de adolescentes e adultos em duas localidades de baixo índice de desenvolvimento humano. Rev Nutr 2009; 22(4):453-465.

33. Oliveira JS, Lira PIC, Maia SR, Sequeira LAS, Amorim RCA, Batista Filho M. Insegurança alimentar e estado nutricional de crianças de Gameleira, zona da mata do Nordeste brasileiro. Rev Bras Saude Matern Infant 2010; 10(2):237-245.

34. Pimentel PG, Sichieri R, Salles-Costa R. Insegurança alimentar, condições socioeconômicas e indicadores antropométricos em crianças em região metropolitana do Rio de Janeiro/Brasil. Rev Bras Est Pop 2009; 26(2):283-294.

35. Motta DG, Peres MTM, Calçada MLM, Vieira CM, Tasca APW, Passarelli C. Consumo alimentar de famílias de baixa renda no município de Piracicaba/SP. Saúde Rev 2004; 6(13):63-70.

36. Silva CCS, Oliveira KBB, Alves AS, Neves JA, Modesto CAC, Vianna RPT. Associação entre consumo alimentar e (in) segurança alimentar e nutricional em São José dos Ramos-PB. Braz J Food Technol 2012; 15(n. esp.):23-30.

37. Bortolini GA, Gubert MB, Santos LMP. Consumo alimentar entre crianças brasileiras com idade de 6 a 59 meses. Cad Saude Publica 2012; 28(9):1759-1771.

38. Maluf RS. Segurança alimentar e fome no Brasil - 10 anos da Cúpula Mundial de Alimentação. Rio de Janeiro: Centro de Segurança Alimentar e Nutricional (CERESAN); 2006. (Relatórios técnicos, n. 2 CERESAN).

Artigo apresentado em 20/11/2016

Aprovado em 01/06/2017

Versão final apresentada em 03/06/2017 